Preparative Free-Flow

Electrophoresis as a

Method of Fractionation of

Natural Organic Materials

GEOLOGICAL SURVEY WATER-SUPPLY PAPER 1817-D

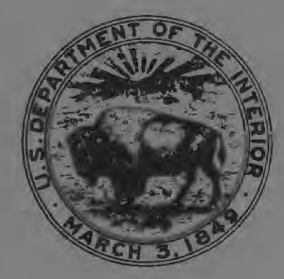




\section{Preparative Free-Flow}

Electrophoresis as a

Method of Fractionation of

Natural Organic Matericls

By J. A. LEENHEER and R. L. MALCOLM

ORGANIG SUBSTANGES IN WATER

GEOLOGICAL SURVEY WATER-SUPPLY PAPER 1817-D

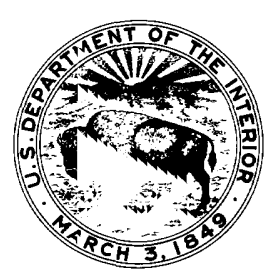




\section{UNITED STATES DEPARTMENT OF THE INTERIOR}

ROGERS C. B. MORTON, Secretary

\section{GEOLOGICAL SURVEY}

V. E. McKelvey, Director

Library of Congress catalog-card No. 73-600376

For sale by the Superintendent of Documents, U.S. Government Printing Office Washington, D.C. 20402 - Price 35c domestic postpaid or 25c GPO Boorstore Stock Number 2401-00293 


\section{CONTENTS}

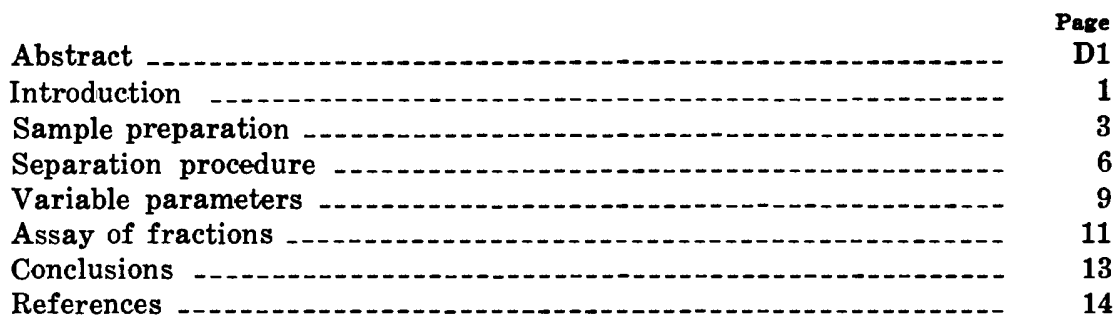

\section{ILLUSTRATIONS}

Figure 1. The Brinkmann Electrophoretic Separator, Model FF4 --

2. Schematic diagram of the buffer flow system of the electrophoretic separator -

3. Electrophoresis and optical density curves of Jane Green Creek organic material 


\title{
PREPARATIVE FREE-FLOW ELECTROPHORESIS AS A METHOD OF FRACTIONATION OF NATURAL ORGANIC MATERIALS
}

\author{
By J. A. Leenheer and R. L. Malcolm
}

\begin{abstract}
Preparative free-flow electrophoresis was found to be an efficient method of conducting large-scale fractionations of the natural organic polyelectrolytes occurring in many surface waters and soils. The method of free-flow electrophoresis obviates. the problem of adsorption upon a supporting medium and permits the use of high potential gradients and currents because of an efficient cooling system. Separations were monitored by determining organic carbon concentration with a dissolved carbon analyzer, and color was measured by absorbance at 400 nanometers. Organic materials from waters and soils were purified by filtration, hydrogen exchange, and dialysis and were concentrated by freeze drying or freeze concentration. In electrophoretic fractionations of natural organic materials typically found in surface waters and soils, color was found to increase with the charge of the fraction.
\end{abstract}

\section{INTRODUCTION}

Electrophoresis has long been recognized to be useful as a method of fractionating complex mixtures of natural and synthetic organic materials. The establishment of an electr: ${ }^{\circ}$ field in a liquid will cause a charged ion in solution or a charged particle in suspension to migrate toward the source of the onposite charge. The rate of electrophoretic migration (electrophoretic mobility) of a charged material in a given liquid depents primarily on two factors: the mass of the material and the magnitude of its electric charge. As the ratio of mass to charge decreases, electrophoretic mobility increases. Fractionat: on of mixtures by electrophoresis depends on taking advantage of the difference in electrophoretic mobilities of the various charged components in the mixture. 
A wide variety of electrophoretic techniques, both theoretical and preparative, exists for quantitatively separating and purifying the individual constituents of complex mixtures (Hannig, 1967). Techniques for theoretical applications include the determination of electrophoretic mobilities, molecular weights, and mass-charge ratios. Preparative electrophoretic methods include moving-boundary, density-gradient, $\mathrm{pH}$-gradient, forced-flow, and continuous-zone electrophoresis. Each of the preparative methods, while employing the principles of electrophoresis, have specific apparatus and applications. Some preparative methods take advantage of multiple principles of separation; for example, densitygradient electrophoresis uses both differences in electrophoretic mobility and specific gravity as means of separation.

Free-flow electrophoresis is one type of continuous-zone electrophoresis. The technique of continuous-zone electrophoresis was first introduced by Grassman and Hannig (1950) and has since become the predominate preparative electrophoretic rethod. In this method, the separation buffer flows in a direction normal to the lines of force of the electric field; a sample is injected at a small spot in the flowing media, and the various components are deflected in different directions depending upon the electrophoretic mobilities of the individual components. The separation buffer in which the sample is carried is usually a solution of weak electrolytes whose primary function is to maintain the electric charge of the components in the sample at a constant level during the electrophoretic fractionation.

Early developments in continuous-zone electrophoresis depended upon the use of a supporting medium for the electrolyte. A variety of supporting mediums such as powdered glass, glass beads, quartz sand, carborudum, and other fine materials were used in enclosed cells. Filter paper, filter cardboard, and fiber glass curtains were the most common means of supporting the electrolyte. Problems involving sample adsorption upon and filtration through the supporting medium, inadequate cooling at high voltage and current, and low sample capacity led to tho development of continuous-zone electrophoresis in free-flowing films of buffer.

Free-flow electrophoresis is carried out in a laminar flow of unsupported electrolyte between glass plates; the absence of a supporting medium nullifies adsorption and filtration interactions, and the free flow allows relatively large amounts of sample to be processed. Free-flow electrophoresis not only fractionates dissolved charged materials, but also has the capability to fractionate 
suspended particulate material on the basis of charge as described by Strickler (1967).

This report deals with the applicability of free-flow electrophoresis as a preparative method for obtaining characterizable isolates from the complex organic mixtures present in surface waters, ground waters, and soil extracts. The investigation presents the optimum procedures for sample preparation, for instrument operation with its variable parameters, and for assays of the fractions. Lastly, speculations on future or potential applications of free-flow electrophoresis is made to aid in the formulation of experiments that can be used to characterize natural organic materials.

\section{SAMPLE PREPARATION}

Before electrophoretic fractionation of a sample is attempted, one should ascertain whether it is possible to use electropl retic fractionation as a means of separation. If separation is pcssible, then the sample should be prepared in such a manner as to optimize the conditions needed for electrophoretic fractionation. Since electrophoresis fractionates electrolytes in aqueous systems, it is necessary that the sample mixture be water soluble or, if particulate, be uniformly dispersible in aqueous systems. Moreover, to fractionate effectively, the sample mixture should rossess components which have differing electrophoretic mobilitios in some given buffer system.

Organic preparations to be used for electrophoretic fractionation should be initially purified from inorganic contaminants. Certain inorganic ions and clay minerals may complex with many organic materials and grossly alter their electrophoretic mobilities and often decrease the resolution of the separation. After initial purification of the sample, inorganic ions and complexing buffers can be added in known amounts to study the effect of complexation upon electrophoresis.

The extraction and purification of dissolved organic material in surface waters was carried out according to the following procedure. The solutions were filtered through a partially clogged membrane filter to separate particulate from dissolved organic material. The next step was the removal of metallic cations by passing the filtrate through a hydrogen-saturated cation-exchange resin. Because, in the waters selected for study, the predominate anions were the organic anions, the removal of the metallic cations essentially desalted the preparation. The water was removed by 
lyophilization, a freeze-dry process which gave a fluff:r, watersoluble organic preparation.

Inorganic anions, which occur in appreciable amounts in certain waters, can be removed after hydrogen saturatior by such methods as dialysis, hydroxide saturation in an anion-exchange column, or gelpermeation filtration. Sample losses are experienced with each of these three methods, however, because it i` difficult to quantitatively separate organic from inorganic anior $\%$ Losses of organic materials in desalting procedures are usually selective for the low-molecular-weight organic materials. The composition of the purified organic material may, therefore, be corciderably different from the composition of the original material. The amount of sample loss can be monitored by dissolved-organiccarbon determinations similar to those run on the electrophoresis separates.

If the sample loss in the desalting procedure is too high to be tolerated, the organic material can be concentrated without drying by freeze concentration. The sample should still be hydrogen saturated, as this prevents precipitation during the concentration process. After freeze concentration, the liquid concentrate can be directly fractionated by free-flow electrophoresis after buffering the material to the correct $\mathrm{pH}$ with the separation buf $э \mathrm{r}$.

The extraction and purification of organic materials from soils and sediments was performed according to the following procedure. The organic matter from soils and sediments was extracted with $0.1 \mathrm{~N} \mathrm{NaOH}$ under nitrogen. The suspended clay was removed by centrifugation and filtration, and the humic-fulvic-acid separation was made on the filtrate acidified to $\mathrm{pH} 1$ with $\mathrm{HCl}$. The humic acid precipitates from the acidified filtrate. and the fulvic acid remains in solution. The $\mathrm{HCl}$ was removed from the fulvic acid by dialysis against distilled water. The final purification of both the humic and fulvic acids was accomplished by hydroxide saturation through an anion-exchange column by eluting with a $\mathrm{NaOH}$ solution at a controlled flow rate at $\mathrm{pH} 9.5$, followed by final hydrogen saturation in a hydrogen-saturated cation-exchange column. The water was removed from the hydrogen-saturated organic matter by means of lyophilization, a process which gives natural organic preparations of low ash content.

Resolubilization of the samples for direct injection into the electrophoresis chamber was accomplished by addition of water to the fulvic acid and the organics extracted from surface waters. The dried humic acids were not soluble upon addition of water. 
They were resolubilized in a minimum amount of $0.1 \mathrm{~N} \mathrm{NaOH}$ solution, and then hydrogen saturated in a cation-exchange column to obtain a water-soluble hydrogen-saturated humic acid.

For preparative fractionations, the organic matter preparations were initially dissolved in water at concentrations ranging from 10 to $20 \mathrm{mg} / \mathrm{ml}$. A $1: 1$ dilution with the separation $k$ uffer gave final concentrations of $5-10 \mathrm{mg} / \mathrm{ml}$. The use of an injected buffer concentration that is half the strength of the separation buffer concentration causes the separation buffer to diffuse into the zone of injection; as it mixes, it concentrates and stabilizes the injected sample band. Care must be taken to maintain the $\mathrm{pH}$ of the separation buffer above $\mathbf{2 . 3}$ for the humic acids; if the $\mathrm{pH}$ is lower, the loss of the negative charge on the humic anior will cause precipitation.

The injected sample solutions should be physically and cl'omically compatible with the separation buffer. If the specific gravity of the injected solution is less than that of the separation buffer, the sample will initially rise in the separation chamber and thus ruin the fractionation. If the buffer concentration of the injected sample is greatly different from the separation buffer concentration, diffusion of the injected buffer will also cause diffusion $\mathrm{cf}$ the sample and reduce resolution of the separation.

For electrophoretic fractionations on which only a diss-lved organic carbon assay of the fractionation is desired, concentrations as low as $50 \mu \mathrm{g} / \mathrm{ml}$ may be used for the injected sample. The dissolved organic carbon is the best parameter for cotermining the relative quantity of organic material in each fraction. As there is roughly a 10 - to $\mathbf{1 0 0 - f o l d ~ d i l u t i o n ~ f a c t o r ~ i n ~ s a m p l e ~}$ concentration from the injected concentration to the peak concentration in the fractionation, the amount of dilution caused by injection of the sample into the separation buffer must be estimated so that the fractions are not too dilute to detect the parameters to be monitored. The dilution factor can be varied by changing the sample-injection and separation-buffer flow rates.

For certain studies, it may be desirable to perform an electrophoretic fractionation on the "raw" sample of water to avoid any alteration of the naturally dissolved organic materials that would occur during preparation for electrophoresis. When th is is done, the buffer electrolyte should be selected to have the same $\mathrm{pH}$ as the native water, and the one chosen should not complex with the organic components to alter their electrophoretic mobility. After the raw sample is diluted in half with the separation buffer prior to injection, the organic carbon concentration must 
still be sufficiently high $(50 \mu \mathrm{g} / \mathrm{ml})$ to permit determination of the organic carbon concentrations in the electrophoretic separates. If the initial sample concentration is too low, it is necessary to use gentle concentration methods such as freeze concentration to increase the sample concentration to levels high enough for electrophoretic fractionation to be performed.

\section{SEPARATION PROCEDURE}

The instrument employeed in this study was the Prinkmann Free-flow Electrophoretic Separator, Model FF4. ${ }^{1}$ A similar instrument and its method of operation has been described by Hannig (1961). The equipment used is shown in figures 1 and 2 . Detailed, stepwise instructions for the operation of the instrument during an electrophoretic separation are given in the instrument manual (Brinkmann Instruments, 1969).

A few additions to and modifications of the operational procedure were found to be useful. A 20-cc pipetting syring? was convenient to use for the filling of the separation chamber with buffer. While the chamber was being filled, operation of the 92-channel peristaltic buffer pump at a slow speed prevented air bubbles from leaking upwards into the separation chamber. After the completion of a run, the separation chamber should ko immediately rinsed out with distilled water. Distilled water can be flushed through the separation chamber by transferring the buffer delivery tubing from the Mariotte flask containing the buffer, to a container of distilled water setting on top of the instrument. The water flows through the separation chamber by gr?vity after opening the 92-channel pump. If water or buffer is rot flowing through the chamber after the voltage is turned off, the cooling capacity remaining in the cooling system may freeze the liquid in the separation chamber and break the glass plates. The water also serves to clean the entire system after each run.

The instrument was found to be virtually mainterance free. The most important point was to thoroughly rinse the separation chamber and the fraction collection tubing with distilled water after each run. If necessary, a 5 percent detergent solution can be used for a rinse. The ion-exchange membranes were changed after every 50 hours of operation to prevent a significant junction potential from developing across the membranes.

1. Use of brand name is for illustrative purpose and does not constitute endorsement by the U.S. Geological Survey. 


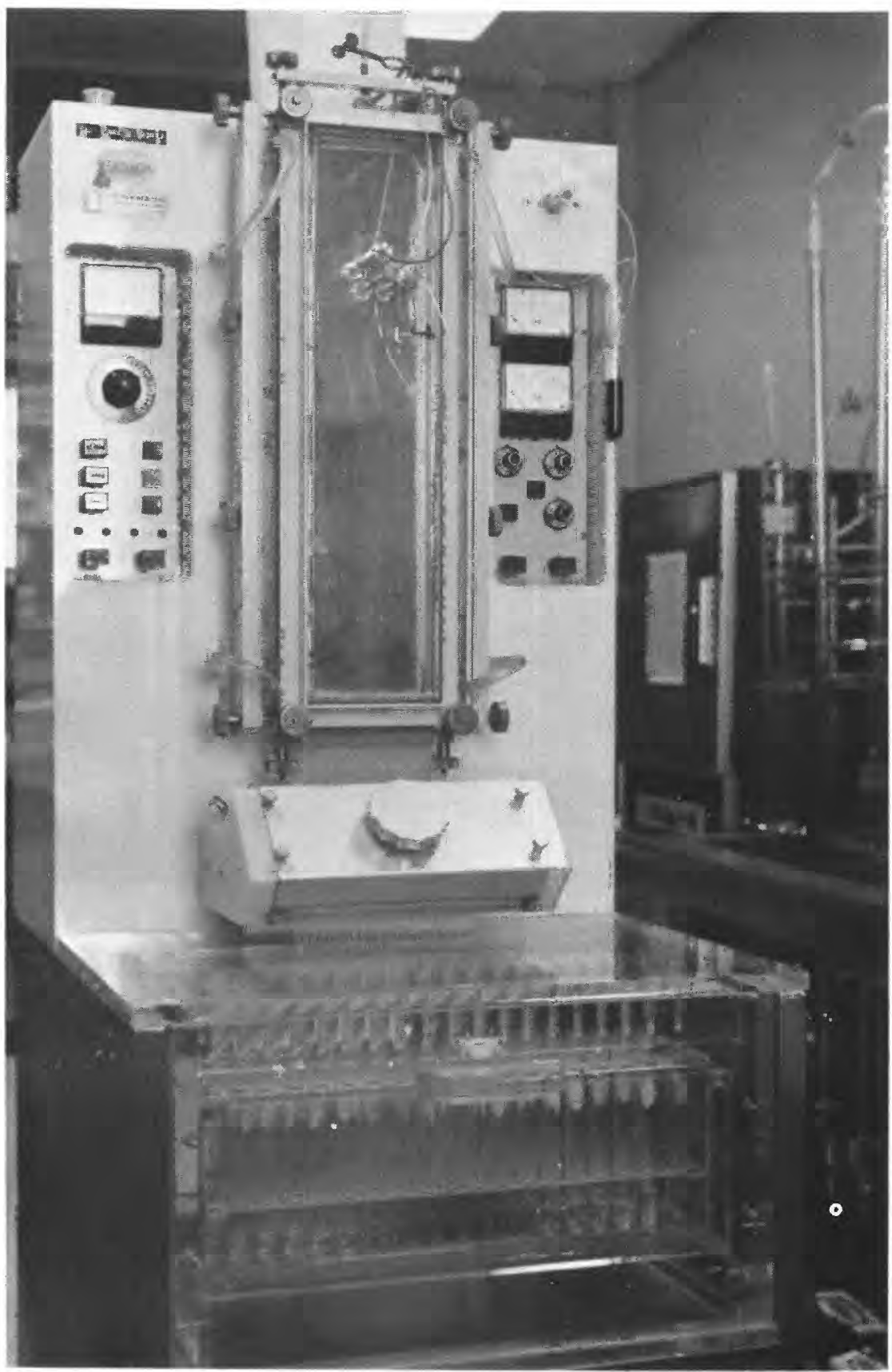

Figure 1.-The Brinkmann Electrophoretic Separator, Model FF4. 


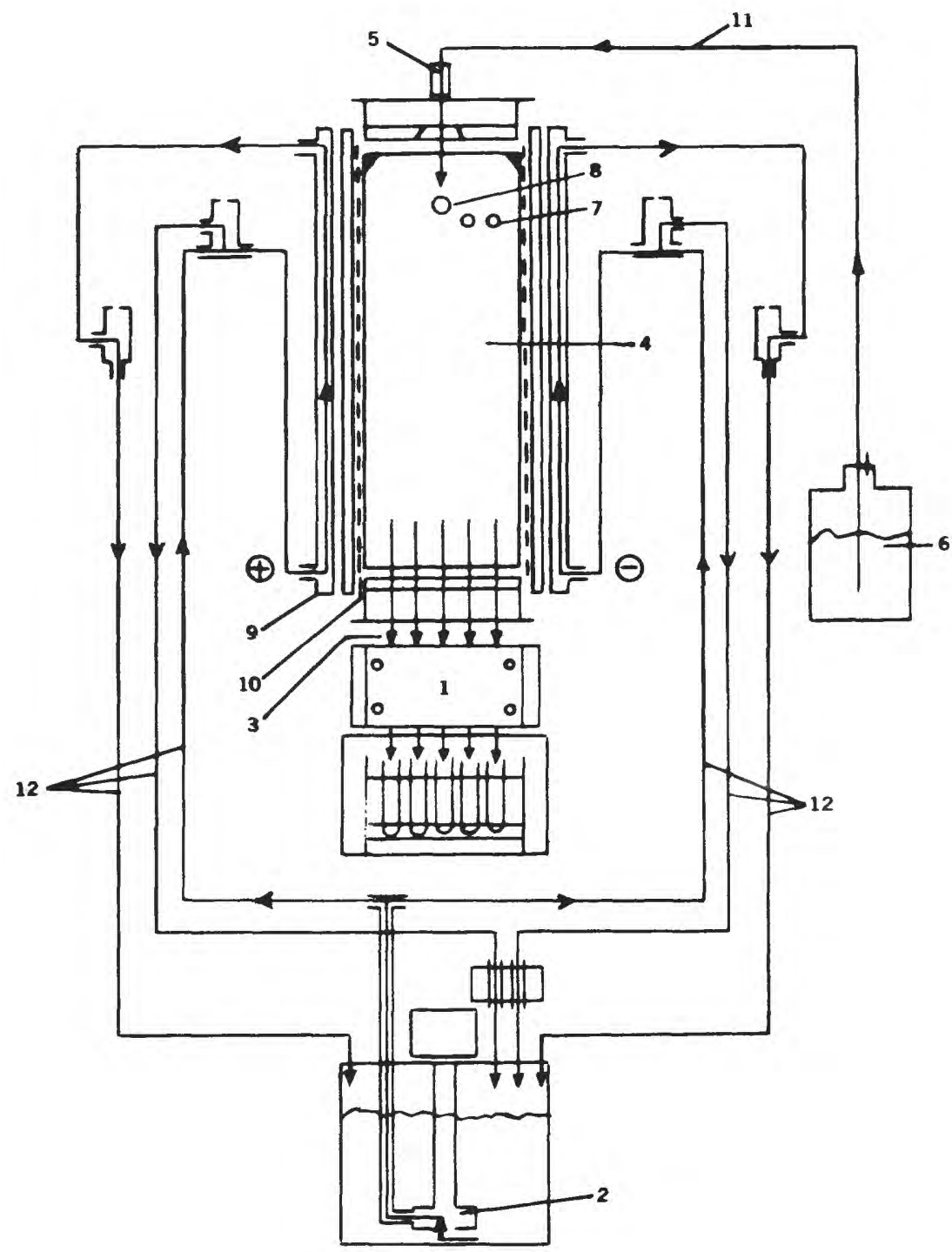

KEY TO SCFEMATIC DIAGRAM

1. 92-channel pump.

2. Electrode rinse pump.

3. $\mathbf{T}$ connector on tube 92 for use in filling separation chamber.

4. Separation chamber.

5. Separation chamber conductivity cell.

6. Mariotte flask reservoir of separation buffer.

7. Sample injection ports.

8. Thermistor port.

9. Electrode rinse chamber.

10. Ion-exchange membrane

11. Separation buffer flow.

12. Electrode rinse buffer flow.

FigURE 2.-The buffer flow system of the electrophoretic separator. 


\section{VARIABLE PARAMETERS}

The variables which determine the resolution and efficier $\mathrm{y}$ of the electrophoretic fractionation are the selection of the $k$ 'uffer, the ionic strength of the buffer, the flow rate of the buffer, the temperature, and the strength of the electric field. These parameters must be optimized for each sample subjected to eloctrophoretic fractionation.

The selection of the electrolyte buffer system is perhars the most important decision for an electrophoretic fractionation. Inorganic buffers must be used if dissolved organic carbon is the parameter used to monitor the fractionation. However, the relatively small ions of inorganic buffers have high migration rates in the electric field of the separation chamber, and the subsequent alteration of the composition and concentration of the buffer system can give gross $\mathrm{pH}$ variation across the electric field. This $\mathrm{pH}$ variation induced by the electric field can be partially remedied by raising the ionic strength of the inorganic buffer. Hovrever, buffer systems of high concentrations give rise to high currents and the associated problems of cooling.

Organic buffer systems, such as tri-citric acid-saccharoseEDTA, possess anions of low electrophoretic mobility; their slow migration permits the maintenance of the buffering capacity in an electric field at relatively low ionic strengths. However, direct monitoring of the organic carbon fractionation is not possible with organic buffers. Spectral properties such as the mersurement of "color" at $400 \mathrm{~nm}$ (nanometers) is often used as a monitor of the fractionation for organic buffer systems.

The buffer system selected for natural organic polyelectrolytes was $0.16 \mathrm{~N}$ phosphoric acid having a $\mathrm{pH}$ of 2.2 This low $\mathrm{pH}$ is near the isoelectric $\mathrm{pH}$ of some of the acids found in natural organic materials. Some of the acids are protonated, and they possess little or no charge; however, other acids are still ionized, and they possess a significant charge. The buffering capacity of this solution was not overcome even after 15 hours of continuous electrophoresis. However, the high conductivity with this buffer of high ionic strength necessitated a fairly low field strencth of 450 volts $(30 \mathrm{v} / \mathrm{cm})$, so that the Peltier cooling system coult dissipate the heat evolved. The electrode rinse buffer was the same concentration as the separation buffer. No advantage could be gained by using an electrode rinse buffer of greater concentration than the separation buffer since minimizing the conductivity 
between the electrodes was more important than oltaining a maximum potential gradient across the separation chamber.

The separation buffer flow rate, which determines the residence time of the sample in the electric field, was approximately 100 $\mathrm{ml} / \mathrm{hr}$. This relatively slow flow rate minimized the dilution of the sample by the buffer, and the resultant lengthy residence time of the sample in the electric field cut down on the requirement for a high field. This slow flow rate and low field strength $(30 \mathrm{v} / \mathrm{cm})$ allowed the fraction of highest mobility to migrate about threequarters the width of the separation chamber. A synthetic dye mixture was used to determine the optimum values of the electric field strength, buffer flow rate, and temperature, on the basis of the mixture's visible separation. The dye mixture graphically demonstrated that $10^{\circ} \mathrm{C}$ was the temperature at which the best resolution was obtained for this system. Higher temperature caused band broadening through thermal diffusion, and lower temperature $\left(5^{\circ} \mathrm{C}\right)$ caused tailing effects in the banc's of high mobility owing to the viscosity of the separation buffor. Lower temperature will also lead to freezing of the buffer solution and possible breakage of the glass plates if the instrument is suddenly shut down when the cooling system is operating at full capacity.

The cooling system consists of 21 Peltier batteries uniformly distributed on a copper plate $8 \mathrm{~mm}$ thick to which the back glass plate of the separation chamber is attached. The batteries act as thermocouples operating in reverse; that is, they either heat or cool depending on the direction of the current flow. The copper plate uniformly distributes the cooling capacities of the Peltier batteries throughout the area of the separation chamler.

The sample was pumped into the separation chamber at the rate of about $8 \mathrm{ml} / \mathrm{hr}$. This rate was sufficiently high to minimize dilution effects, yet not so high as to lead to band broadening at the point of injection.

The operating conditions outlined above were designed to effect an electrophoretic separation based on isoelectric $\mathrm{pH}$ differences of the natural organic acids. Electrophoretic separations based on buffer complexation and exchange with the sample to alter the electrophoretic mobility of the sample have also been employed. Clapp (1957) used a borate buffer which complexed with the polysaccharides in a soil water extract giving the polysaccharides an increased negative charge. Park and Lewis (1969) found that addition of $\mathrm{Th}^{+4}$ to a $\mathrm{Na}_{2} \mathrm{CO}_{3}$ buffer decreased the charge on illite and kaolinite through ion exchange without appreciably affecting 
the charge on montmorillionite in a free-flow electropl oretic separation of clay minerals. Because the method of free-flow electrophoresis is able to process large amounts of material, multiple electrophoresis can be performed on a sample under a variety of operating conditions.

\section{ASSAY OF FRACTIONS}

Electrophoretic fractionation as well as every other system of fractionation needs fast and accurate methods of monitoring the separation. The basic fractionation of the organic material was monitored by a dissolved-carbon analyzer. The analyzer combusts the dissolved sample on a platinum catalyst at $950^{\circ} \mathrm{C}$, and the amount of $\mathrm{CO}_{2}$ produced is measured in a gas cell by an infrared analyzer. Individual determinations of the total organic carbon concentration in a certain fraction can be made in 100 seconds on only $40 \mu \mathrm{l}$ of sample. For the phosphoric acid buffer at $\mathrm{pH} 2.2$, there are no carbonates present to interfere with the organic carbon determination, and concentrations down to about $1 \mathrm{ppm}$ can be accurately determined. For buffer systems that onerate above $\mathrm{pH} \mathrm{4}$, inorganic carbon will be present as carbonates and bicarbonates, and organic carbon must be determined as th $\circ$ difference between total carbon and inorganic carbon. In $\mathrm{e}^{\mathbf{l} e c t r o-}$ phoretic fractionation of organic materials, organic carbon should be monitored, because some of the organic components are likely to be colorless and undetectable by conventional monitoring: techniques. Index of refraction and ultraviolet absorption can also be used to detect colorless organic components.

Figure 3 presents an example of an electrophoretic fractionation of the organic material extracted from Jane Greene Creek near Melbourne, Fla. The organic carbon curve shows two distinct fractions; a slightly charged fraction peaking in tube 26 , and a highly charged fraction peaking in tube 46 . However, conventional monitoring of "color" at $400 \mathrm{~nm}$ only detects the charged component with maxima at tube 52.

Other spectral methods suitable for analysis without removal of the buffer solution include fluorescence, ultraviolet, and index of refraction. Tests for various functional groups on a semiquantitative basis can also be used to monitor the fractionation. Wildenhain and Henseke (1966) used a number of colorimetric spray reagents to test for the presence of carbonyl, carboxyl, aliphatic and phenolic hydroxyl, and amino groups in fulvic-acids seprrates. Sugars can be colorimetrically determined with $\alpha$-naphthol, and 


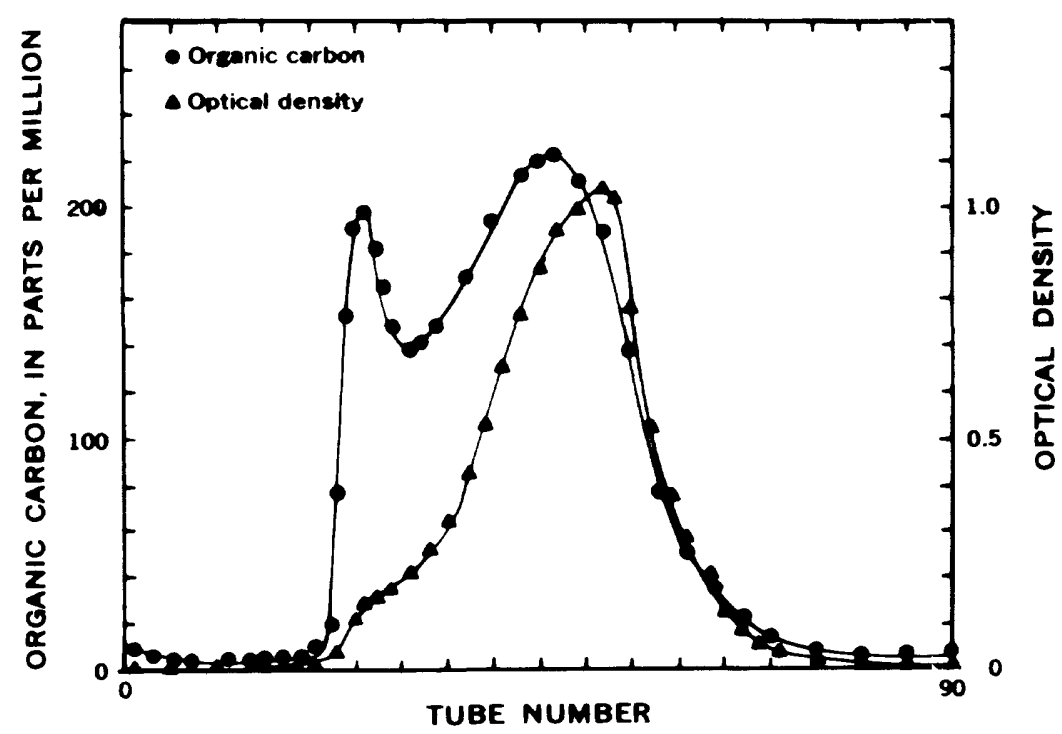

FIGURE 3.-Electrophoresis and optical density curves of Jare Greene Creek organic material.

uronic acids with a carbazole method (Department of Agronomy, 1956). Total nitrogen is best determined by a microcoulometric titration; however, nesslerization or the Kjeldahl method can also be used with less sensitivity and speed.

A more exhaustive study of the products of the electrophoretic fractionation requires that the sample be reisolated from the buffer in which it is dissolved. The phosphoric-acid buffer was removed from solution by simple dialysis against deiorized water for a period of 2 days. Gelpermeation filtration and ion exchange are other alternative methods of desalting; however, each method will cause losses of organic material, and the method chosen must fit the needs of the experimenter. After desalting and hydrogen saturation by a cation-exchange resin, the samples were lyophil- 
ized to give fluffy, water-soluble preparations of low ash content suitable for analysis.

For functional group assays of electrophoretic fractionation of organic materials, it is essential to obtain infrared spectra of the separates. The spectra should be run on the fractions of highest purity since inorganic components arising from the basic preparation or from an inorganic buffer which was not remored can seriously mask certain portions of the infrared spectra. It was found that the resolution and character present in the infrared spectra was much more detailed for the separates after fractionation than for the gross mixture prior to electrophoresis. Elamental analysis for carbon, hydrogen, oxygen, nitrogen, sulfur, and phosphorus should be run on the freeze-dried preparations to give empirical compositions which are often indicative of the chemical composition of the various components. Titration curves, both aqueous and nonaqueous, can be used to give equivalent weights; and specific ion electrodes can be used to investigate erchange phenomena and determine stability constants for various metalorganic complexes. If the separates can be volatilized either directly or through the preparation of derivatives, then gas chromatography (GC), mass spectroscopy (MS), or GC-MS comkination can be powerful tools to completely characterize the separates.

\section{CONCLUSIONS}

Free-flow electrophoresis presents the researcher with a powerful new method in the field of separation science. Natural organic materials found in soils and surface waters have been very resistant to separation and purification by conventional techniques such as selective extraction, distillation, and recrystall:ation. Preparative electrophoresis, which has long shown promise in separating organic mixtures on the basis of charge, has come of age in the development of the free-flow system which is able to process and fractionate the large amounts of material neeted for extensive characterization of the complex mixture found in natural organic materials. It is hoped that once the characteristic electrophoretic separates have been identified, it may be possible for natural organic systems to be characterized chemically to a limited 
extent, simply by their electrophoretic carbon curves. Identified compounds should repeatedly appear in the same position in electrophoresis curves regardless of their origin.

\section{REFERENCES}

Brinkmann Instruments, 1969, Electrophoretic separator model FF4 instrumental manual: Westbury, N.Y., 17 p.

Clapp, C. E., Jr., 1957, High molecular weight water-soluble muck; isolation and determination of constituent sugars of a borate complex-forming polysaccharide employing electrophoretic techniques: Ph.D. dissertation. Abs. 17 , p. $963-964$.

Department of Agronomy, 1956, Organic Soils Laboratory, Chemical Methods: Cornell University.

Grassman, W., and Hannig, K., 1950, Simple process for contintous separation of mixtures on filter paper by electrophoresis: Naturwis venschaften, v. 37 ; p. 397-402.

Hannig, K., 1961, Carrier-free continuous electrophoresis ard its use: Zeitschr. Anal. Chemie, v. 181, p. 244-254.

1967, Preparative electrophoresis, in M. Bier, ed., Electrophoresis: Theory, Method, and Applications: New York, Academic Press, p. 423-465.

Park, R. G., and Lewis, G. C., 1969, Electrophoretic separation and fractionation of clay mixtures: Am. Mineralogist, v. 54, p. $147 \AA-1476$.

Strickler, A., 1967, Continuous particle electrophoresis; a nev analytical and preparative capability: Separation Science, v. 2, p. 33F-355.

Wildenhain, W., and Henseke, G., 1966, Fractionation of fulvic acid on Sephadex and characterization through color reactions: Zeitschr. Anal. Chemie, v. 229, p. 271-281. 